\section{Le placebo à l'hôpital \\ Regard sur son utilisation dans les services de médecine polyvalente}

\section{Anne Héron ${ }^{1,2}$, Christophe Leroux ${ }^{3}$, David Dubayle ${ }^{4}$}

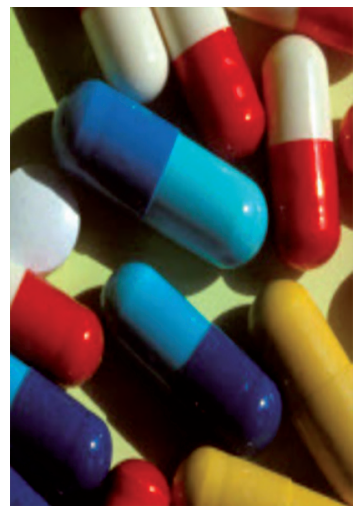

${ }^{1}$ Faculté de Santé, Université de Paris, 4, avenue de l'Observatoire, 75006 Paris, France.

${ }^{2} U$ nité de recherche clinique URC28, Groupement des Hôpitaux Publics Euréliens, Dreux, France. ${ }^{3}$ Fédération francophone de médecine polyvalente, Unité de médecine polyvalente, Centre hospitalier Victor Jousselin, 44, avenue J.F. Kennedy, 28100 Dreux, France. ${ }^{4}$ UFR Biomédicales, CNRS

actif, qui est administré dans le but d'être agréable au patient (du latin placebo, «je plairai »), et qui est susceptible d'améliorer les sympUMR8002, Université de Paris, 45 , rue des Saints Pères, 75006 Paris, France. anne.heron@parisdescartes.fr professionnels «croit» en l'effet placebo, mais se considèrent cependant insuffisamment informés et formés à son utilisation. Fréquemment utilisé à l'hôpital, son efficacité thérapeutique étant largement admise, des considérations éthiques et juridiques imposent de recommander que sa nature soit précisée au patient lors de sa prescription (au même titre que les autres préparations hospitalières). On parlera alors de «placebo ouvert», c'est-à-dire que des explications sur les effets et les mécanismes d'action du placebo seront données au patient. Des études récentes montrent que dire au patient qu'il reçoit un placebo ouvert n'affecte en rien son effet. <

\title{
Le placebo
}

\section{De quoi s'agit-il ?}

Un traitement placebo est défini comme un traitement ne contenant pas de composé pharmacologiquement

Vignette (Photo @ Inserm - Michel Depardieu).

sont dépourvus de composés actifs et spécifiques (par exemple des gélules de cellulose distribuées par la pharmacie hospitalière). Ils se distinguent des placebos dits impurs qui correspondent à des produits actifs sur certaines pathologies mais dont l'usage est détourné pour traiter un symptôme non ciblé par le principe actif du médicament comme, par exemple, la vitamine $D$ en cas de fatigue.

L'administration d'un placebo peut entraîner l'apparition d'effets regroupés sous le terme d' «effets nocebo » $[1,2]$, qui correspondent à l'aggravation des symptômes cliniques ou à la survenue d'effets indésirables. Les effets les plus fréquemment observés sont une somnolence, des céphalées, des troubles de la concentration, des maux de ventre, une diarrhée, des dermatoses et/ou une bouche sèche. Ils concernent environ un quart des patients traités [3], ce qui est à prendre en considération lors de la prescription.

Les traitements placebo jouent un rôle important en médecine dans deux domaines principaux : les études expérimentales, dans lesquelles ils sont utilisés comme contrôle pour déterminer les effets spécifiques d'un nouveau traitement (par exemple dans des essais cliniques en double aveugle); en pratique clinique, où ils sont considérés comme de véritables outils thérapeutiques.

\section{Mode d'action du placebo}

Le placebo agit via l'effet dit placebo, un effet étudié depuis les années $1950[4,5]$. L'effet placebo peut se définir comme la différence entre 
l'effet observé (lors de l'administration d'un médicament) et celui attribuable à l'action pharmacologique d'un médicament. II est donc le résultat de la différence entre l'effet thérapeutique global (cliniquement mesurable) et l'effet spécifique (c'est-à-dire l'effet pharmacodynamique du médicament). Cette définition devrait cependant considérer le fait que, parfois, ce que l'on suppose être un effet placebo ou un effet thérapeutique, n'est finalement que le résultat de l'évolution naturelle de la maladie ou des symptômes. La réponse au placebo est empreinte d'un certain mystère [6]: elle varie d'un individu à l'autre et, chez un même patient, d'une prescription sur l'autre. De nombreuses études montrent que la plage d'efficacité du placebo peut s'étendre de 4 à $86 \%$ selon les pathologies, avec des résultats qui sont importants dans le traitement de la douleur ou des nausées [7]. La réponse au placebo est également influencée par le contexte environnemental, la culture, la maladie, et dépend du pouvoir de conviction du médecin, de son empathie mais aussi de la confiance que lui accorde le patient [8-11]. L'effet placebo est également attribué à l'effet symbolique du médicament, par opposition à ses propres effets pharmacologiques ou physiologiques [12]. D'autres paramètres interviennent dans l'efficacité d'un placebo, comme sa voie d'administration, sa posologie, sa forme galénique mais également sa couleur. Au plan symbolique, les couleurs sont en effet mentalement associées à des significations particulières : les couleurs froides, telles que le bleu, sont associées au calme, les couleurs chaudes, comme le rouge, sont stimulantes. La galénique utilise cette symbolique des couleurs. Ainsi, les hypnotiques sont des cachets blancs ou bleus alors que les psychostimulants sont très souvent de couleur rouge. Ce code couleur est conservé lors de la fabrication des placebos. Une meilleure efficacité d'un traitement placebo a également été constatée lorsque le patient était impliqué dans la séquence thérapeutique : compter méticuleusement les gouttes d'un traitement augmente son effet. Plus le traitement est douloureux (par exemple, voie sous-cutanée versus voie orale dans le cas du traitement de la migraine), plus l'effet placebo sera important [13]. Les patients ont en effet de plus grandes attentes vis-à-vis des traitements douloureux car ils sont supposés être plus élaborés [14]. Le patient se sent, dans ce cas, d'avantage considéré, le niveau d'attention et le contact physique des praticiens étant plus importants [15].

La réponse au placebo n'est pas seulement psychologique. Elle fait partie d'une réalité biologique. Il s'agit de processus cérébraux entraînant des modifications importantes de paramètres physiologiques, telles que la libération d'hormones et de neurotransmetteurs, la réponse du système immunitaire, la modification des paramètres cardiovasculaires, la modulation de la sensibilité à la douleur, etc. [16].

\section{Comment utiliser au mieux un placebo?}

L'efficacité d'un placebo dépendrait de la manière dont sont informés les patients qui le reçoivent. Dire aux patients qu'ils reçoivent un traitement analgésique conduirait, par exemple, à une libération intracérébrale d'endorphines [17]. Dans une étude récente, une stimulation douloureuse sur le bras de volontaires s'est vue soulagée par un placebo alors qu'ils pensaient avoir reçu un médicament. Quand ils ont appris, après plusieurs jours, qu'il s'agissait d'un placebo, l'effet analgésique a toutefois persisté [18]. Ces observations renforcent deux mécanismes psycho-physiologiques pouvant expliquer l'effet placebo : la suggestion et le conditionnement [2]. Des neurotransmetteurs, tels que la dopamine et la sérotonine, seraient impliqués dans le mécanisme d'action de l'effet placebo [19]. Des chercheurs du centre médical Beath Israel Deaconess (BIDMC) et de l'université de Harvard ont récemment avancé I'hypothèse d'un composant génétique dans l'effet placebo, en montrant l'implication des allèles du gène COMT (catéchol-0-méthyltransférase) responsable de la synthèse de la dopamine cérébrale [20]. L'élévation dopaminergique pourrait ainsi être l'un des substrats biologiques de l'effet placebo activant la voie de la récompense «motivation-émotion». Malgré la reconnaissance de son existence, il n'existe que très peu d'études portant sur l'utilisation du placebo en tant qu'outil thérapeutique dans la pratique de la médecine courante et, en dehors des essais cliniques, elle reste sujette à controverse sur les plans éthique, professionnel et juridique [21].

L'administration d'un placebo, en substitution d'un traitement dont l'efficacité thérapeutique est prouvée, peut priver le patient d'une chance d'amélioration clinique. Il ne doit donc pas être utilisé pour le traitement de pathologies graves, telles que le cancer, et doit être réservé à l'amélioration du confort du patient. C'est aussi le cas pour certains traitements alternatifs, qui peuvent avoir des effets délétères lorsque les patients se privent du traitement adéquat préconisé par les cliniciens, alors que celui-ci est efficace sur la disparition des symptômes $[22,38](\rightarrow)$.

$\rightarrow$ Voir le Forum de B. Jordan, $m / s n^{\circ} 11$, novembre 2018, page 999

De même, chez l'enfant, la majorité des infections respiratoires aiguës sont d'origine virale. Elles s'améliorent généralement sans traitement.

Peu de données existent concernant la fréquence et les circonstances de l'utilisation du placebo en pratique clinique. Pourtant, chaque clinicien a dû utiliser, un jour, un traitement placebo, ou a assisté à son utilisation au sein de l'hôpital. Les pharmacies hospitalières distribuent chaque année des dizaines de milliers de gélules contenant un placebo, sans qu'aucune estimation de la consommation de ce type de traitement n'ait été réalisée au niveau national. Ce sujet est peu abordé dans la littérature scientifique et au sein même des institutions de soins. Pourtant, selon une étude américaine réalisée en 2008 [23], $45 \%$ des médecins hospitaliers américains disent prescrire des placebos à leurs patients. Qu'en est-il alors de la France? À notre connais- 
sance, seule une étude portant sur leur utilisation dans les services de rhumatologie a été publiée depuis 2001 [24].

\section{Le placebo en milieu hospitalier}

\section{Une enquête nationale de santé publique}

La médecine polyvalente se développe dans de nombreux hôpitaux publics et privés et tend à devenir le principal service, en aval des services d'urgence. Elle représente un interlocuteur hospitalier direct et un remède pour la médecine libérale des territoires, en particulier pour la prise en charge globale des patients poly-pathologiques. L'équipe pluridisciplinaire de médecine polyvalente traite en effet un large éventail de pathologies, en particulier lorsqu'elles sont complexes et impliquent les principales disciplines médicales liées à la cardiologie, la pneumologie, la gastro-entérologie, l'endocrinologie, l'uro-néphrologie, la rhumatologie, la gériatrie, l'infectiologie, etc. Compte tenu de la composante multifactorielle de ces pathologies et de la complexité des soins qu'elles nécessitent, les professionnels se sentant parfois démunis peuvent utiliser un placebo. Nous montrons, à l'issue de notre enquête, que le placebo est effectivement utilisé à l'hôpital par une majorité de professionnels de santé des services de médecine polyvalente.

\section{Le placebo en médecine polyvalente}

L'enquête que nous avons réalisée s'adressait aux professionnels de santé pratiquant la médecine polyvalente dans les hôpitaux publics de France métropolitaine (Tableau I). La dénomination des services contactés était: Service de médecine polyvalente (pour les services portant déjà ce titre), Service de médecine interne, Service de médecine générale, Service de médecine interne et polyvalente ou Service de médecine interne et générale pour ceux qui peuvent être assimilés à un service de médecine polyvalente car ils ont des activités de soins similaires.

Trois cent soixante-douze questionnaires ont été transmis à ces services, invitant l'ensemble du personnel médical et paramédical à y répondre. Les professionnels ont également eu la possibilité de remplir ce questionnaire en ligne via un lien Internet sur le site du premier congrès organisé par la Fédération française de médecine polyvalente, devenue depuis peu la Fédération francophone de médecine polyvalente. Le questionnaire comportait 15 points et concernait le statut du professionnel, les types de placebos utilisés, les modes et les circonstances de leur utilisation, ainsi que l'opinion du professionnel sur le placebo et son effet. Le questionnaire pouvait être rempli anonymement en moins de 10 minutes. Les professionnels ont eu trois mois pour répondre.

Cent cinquante-trois réponses ont été reçues (120 par courrier et 33 complétées directement en ligne) (soit un taux de réponse de $41 \%$ ). Les professionnels de santé interrogés étaient des médecins ( $55 \%$ ), des infirmiers(ères) (27\%), des aides-soignants(es) (9\%), des cadres de santé ( $5 \%$ ) ou des étudiants(es) en médecine (4\%) (Tableau II). Leur âge moyen était de $41,8 \pm 10,3$ ans (21-67 ans) et $62 \%$ étaient des femmes.

L'analyse des réponses reçues montre qu'en moyenne, $65 \%$ des professionnels utilisent des placebos (78\% des infirmièr(e)s, $62 \%$ des médecins, $67 \%$ des internes, $75 \%$ des cadres et $43 \%$ des aides- soignant(e)s ayant répondu à l'enquête, ont déclaré utiliser des placebos). Ils sont principalement administrés en cas d'insomnie ( $63 \%$ ), de douleur ( $54 \%$ ) et/ou d'anxiété (48\%) (Figure 1A - Histogramme de gauche) et sont utilisés dans diverses circonstances: «pour obtenir un effet placebo » $(19 \%)$, «chez les patients dits difficiles » c'est-à-dire solliciteurs d'attention et de soin ( $16 \%$ ) ou «pour rassurer les patients » (14\%) (Figure IA - Diagramme circulaire de droite). La voie d'administration la plus courante est la voie orale $(71 \%)$ - plus particulièrement sous la forme de gélule (61\%) - la voie injectable représente $6 \%$ des cas. Les couleurs dominantes de ces gélules, librement choisies par les professionnels en fonction du contexte thérapeutique, sont le rouge ( $26 \%$ ), le blanc (23\%) ou le bleu (16\%). Les placebos sont le plus souvent administrés la nuit, dans $65 \%$ des cas (Figure 1B) et suivent une ordonnance médicale (58\% des cas) (Figure 1C). Le patient n'est presque jamais informé qu'il reçoit un placebo (86\%) (Figure 1D).

La majorité des professionnels qui ont participé à l'étude, qu'ils soient utilisateurs (61\%) ou non utilisateurs ( $51 \%$ ) de placebo, considèrent qu'il représente un traitement efficace (Figure $2 A$ ). Plus de la moitié des utilisateurs pensent que donner un placebo n'influence pas la relation entre soignant et soigné, alors que les non-utilisateurs sont plus partagés (une différence significative existe entre les deux groupes) (Figure 2B). En revanche, une grande majorité des professionnels, qu'ils soient utilisateurs ou non, se considèrent insuffisamment informés ou formés à l'utilisation des placebos (69\%) (Figure 2C) et $53 \%$ ne connaissent pas les effets nocebo. En revanche, une grande proportion de professionnels «croient » en l'effet placebo (87\%) (Figure 2D).

\section{Les indications et les formes galéniques de placebos utilisés en médecine polyvalente}

L'utilisation du placebo est donc fréquente dans les hôpitaux français puisque près des deux tiers des professionnels de médecine polyvalente ayant répondu à l'enquête ont déclaré l'utiliser. La forme orale, en gélule rouge, est la forme d'administration la plus couramment utilisée. Dans une revue systématique internationale réalisée à partir de 22 études issues de 12 pays différents, il est montré que la proportion de médecins déclarant utiliser souvent ou périodiquement un placebo varie entre $17 \%$ et $80 \%$ pour les placebos purs, entre $54 \%$ et $57 \%$ pour les placebos impurs et entre $41 \%$ et $99 \%$ pour les deux types de placebos [25]. La France ne fait donc pas exception. Les professionnels de médecine polyvalente déclarent utiliser des placebos 


\begin{tabular}{|c|c|c|c|c|}
\hline Régions & Départements & $\begin{array}{c}\text { Nombre } \\
\text { de services }\end{array}$ & Départements & $\begin{array}{c}\text { Nombre } \\
\text { de services }\end{array}$ \\
\hline \multirow{7}{*}{$\begin{array}{l}\text { Auvergne-Rhône-Alpes } \\
\qquad \mathrm{N}=53\end{array}$} & Ain & 9 & Haute-Savoie & 2 \\
\hline & Allier & 4 & Isère & 6 \\
\hline & Ardèche & 5 & Loire & 2 \\
\hline & Cantal & 5 & Puy-de-Dôme & 6 \\
\hline & Drôme & 3 & Rhône & 2 \\
\hline & Haute-Loire & 4 & Savoie & 5 \\
\hline & Côte-d'Or & 5 & Nièvre & 6 \\
\hline \multirow{3}{*}{$\begin{array}{l}\text { Bourgogne-Franche-Comté } \\
\qquad N=27\end{array}$} & Doubs & 1 & Saône-et-Loire & 2 \\
\hline & Haute-Saône & 5 & Yonne & 4 \\
\hline & Jura & 4 & & \\
\hline \multirow{5}{*}{$\begin{array}{l}\text { Centre-Val-de-Loire } \\
\qquad N=17\end{array}$} & Côtes-d'Armor & 5 & Ille-et-Vilaine & 3 \\
\hline & Finistère & 8 & Morbihan & 7 \\
\hline & Cher & 3 & Indre-et-Loire & 3 \\
\hline & Eure-et-Loir & 4 & Loir-et-Cher & 2 \\
\hline & Indre & 3 & Loiret & 2 \\
\hline $\begin{array}{l}\text { Corse } \\
\mathrm{N}=6\end{array}$ & Corse-du-Sud & 5 & Haute-Corse & 1 \\
\hline \multirow{5}{*}{$\begin{array}{l}\text { Grand-Est } \\
N=39\end{array}$} & Ardennes & 4 & Marne & 3 \\
\hline & Aube & 3 & Meurthe-et-Moselle & 4 \\
\hline & Bas-Rhin & 5 & Meuse & 2 \\
\hline & Haut-Rhin & 4 & Moselle & 5 \\
\hline & Haute-Marne & 3 & Vosges & 6 \\
\hline \multirow{3}{*}{$\begin{array}{l}\text { Hauts de France } \\
\qquad \mathrm{N}=38\end{array}$} & Aisne & 8 & Pas-de-Calais & 5 \\
\hline & Nord & 13 & Somme & 8 \\
\hline & Oise & 4 & Seine-Saint-Denis & 1 \\
\hline \multirow{3}{*}{$\begin{array}{l}\text { Ile-de-France } \\
\qquad N=25\end{array}$} & Essonne & 5 & Val d'Oise & 6 \\
\hline & Hauts-de-Seine & 4 & Yvelines & 3 \\
\hline & Seine-et-Marne & 6 & & \\
\hline \multirow{3}{*}{$\begin{array}{l}\text { Normandie } \\
\qquad \mathbf{N}=\mathbf{3 0}\end{array}$} & Calvados & 6 & Orne & 9 \\
\hline & Eure & 6 & Seine-Maritime & 7 \\
\hline & Manche & 2 & & \\
\hline \multirow{6}{*}{$\begin{array}{l}\text { Nouvelle-Aquitaine } \\
\qquad \mathrm{N}=37\end{array}$} & Charente & 3 & Gironde & 6 \\
\hline & Charente-Maritime & 5 & Haute-Vienne & 1 \\
\hline & Corrèze & 2 & Landes & 2 \\
\hline & Creuse & 2 & Lot-et-Garonne & 4 \\
\hline & Deux-Sèvres & 6 & Pyrénées-Atlantiques & 2 \\
\hline & Dordogne & 2 & Vienne & 2 \\
\hline \multirow{6}{*}{$\begin{array}{c}\text { Occitanie } \\
\mathrm{N}=35\end{array}$} & Ariège & 3 & Hérault & 3 \\
\hline & Aude & 5 & Lot & 3 \\
\hline & Aveyron & 7 & Lozère & 1 \\
\hline & Gard & 4 & Pyrénées-0rientales & 1 \\
\hline & Gers & 1 & Tarn & 3 \\
\hline & Hautes-Pyrénées & 3 & Tarn-et-Garonne & 1 \\
\hline \multirow{3}{*}{$\begin{array}{l}\text { Pays-de-Loire } \\
\qquad N=18\end{array}$} & Loire-Atlantique & 2 & Sarthe & 5 \\
\hline & Maine-et-Loire & 2 & Vendée & 7 \\
\hline & Mayenne & 2 & & \\
\hline \multirow{3}{*}{$\begin{array}{l}\text { Provence-Alpes-Côte-D'azur } \\
\qquad \text { N = } 24\end{array}$} & $\begin{array}{l}\text { Alpes-de-Haute-Pro- } \\
\text { vence }\end{array}$ & 2 & Hautes-Alpes & 3 \\
\hline & Alpes-Maritimes & 3 & Var & 7 \\
\hline & Bouches-du-Rhône & 6 & Vaucluse & 3 \\
\hline
\end{tabular}

Tableau I. Régions, départements et nombre de services inclus dans l'étude. 
A Circonstances d'utilisation des placebos

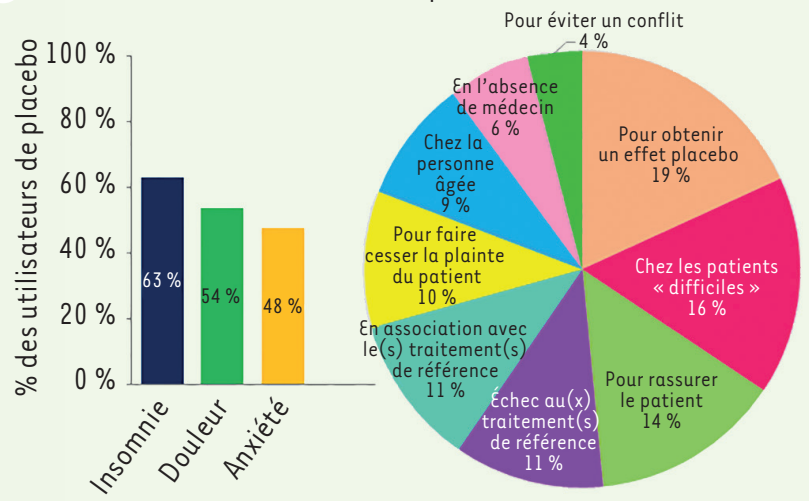

C $100 \%$ Le recours à un placebo fait-il suite à une prescritpion?

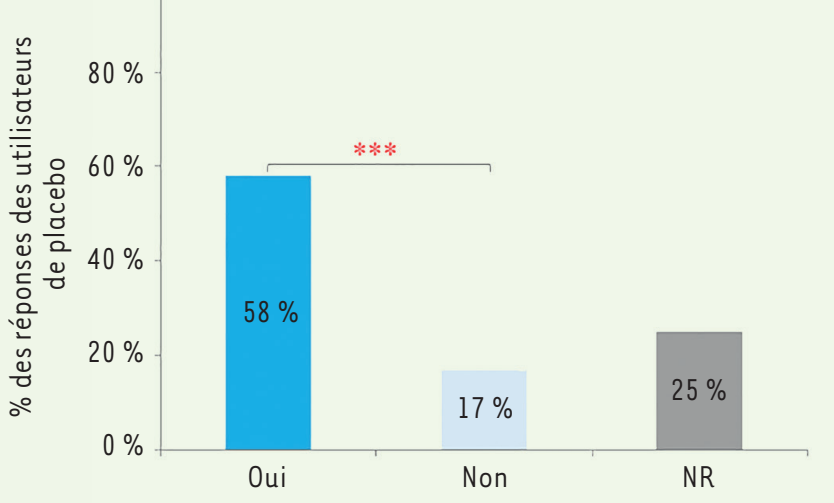

B $\quad 100 \%$ À quel moment le patient reçoit-il le placebo?
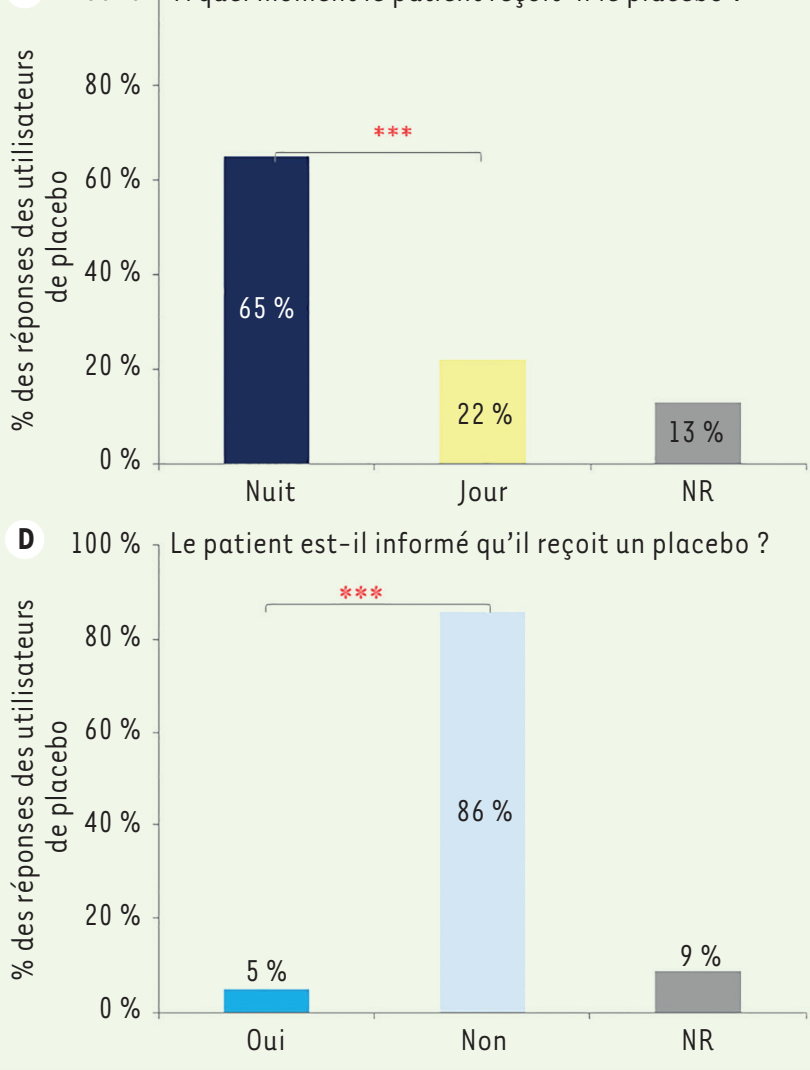

Figure 1. Réponses des professionnels de santé déclarant utiliser des placebos dans des unités de médecine polyvalente. A. Les professionnels utilisent les placebos dans diverses circonstances : insomnie, douleur et anxiété (histogramme), pour obtenir un effet placebo chez les patients difficiles ou pour rassurer le patient (diagramme circulaire). B. L'histogramme illustre le moment où le patient reçoit le placebo (le plus souvent la nuit). C. Histogramme révélant que le patient reçoit le placebo majoritairement après prescription médicale. $D$. Les patients ne sont presque jamais informés qu'ils reçoivent un placebo. ${ }^{*} \star_{p}<0,001$ : degré de significativité des différences inter-groupes. NR : non renseigné.

pour traiter l'insomnie, la douleur et l'anxiété, ce qui est confirmé pour d'autres spécialités médicales $[7,10,25-27](\rightarrow)$.

La forme la plus fréquemment utilisée est une de F. Haour, $m / s$ $n^{\circ} 3$, mars 2005, page 315 $(\rightarrow)$ Voir la Synthèse

gélule rouge, administrée par voie orale. La couleur, la taille, la forme, la voie d'administration et les associations de placebos entre eux [2831] peuvent influencer l'effet du placebo. Le placebo étant utilisé le plus souvent à des fins analgésiques, il n'est pas surprenant que les professionnels de médecine polyvalente préfèrent la couleur rouge, symboliquement associée à la douleur. Bien que plus invasive, la voie injectable, considérée comme la voie d'administration la plus efficace $[32,33]$, est utilisée par certains professionnels, alors que le rapport bénéfice/risque ne favorise pas cette voie. Elle ne devrait donc être utilisée que dans de rares circonstances qui restent à préciser.

En médecine polyvalente, un placebo est principalement prescrit la nuit, en réponse à des symptômes (douleur, anxiété et insomnie) qui sont amplifiés à ce moment, mais qui ne nécessitent généralement pas de diagnostic médical d'urgence. Les professionnels de santé, en nombre limité la nuit, utilisent ainsi un placebo afin d'apporter un confort au patient. Mais alors pourquoi ne pas, dans ces cas, administrer un traitement pharmacologique contenant un principe actif?

«Mettre un terme aux plaintes des patients dits 'difficiles' ». Malgré les avancées qui ont été réalisées sur la prise en charge de la douleur, de l'anxiété, l'évaluation reste difficile et subjective et dépend de la sensibilité et de la disponibilité des professionnels. Tous savent ce que recouvrent les termes de patients «difficiles». Dans la littérature internationale, ces patients peuvent être comparés aux «patients qui exigent trop de médicaments », ce qui concerne en fait des patients solliciteurs d'attention et de soins [25].

\section{Le placebo : un traitement miracle?}

L'utilisation du placebo répond à un réel besoin des professionnels afin d'optimiser le confort du patient, de le rassurer, voire de trouver une solution alternative en cas d'échec 
A Considérez-vous qu'un placebo soit un traitement efficace?

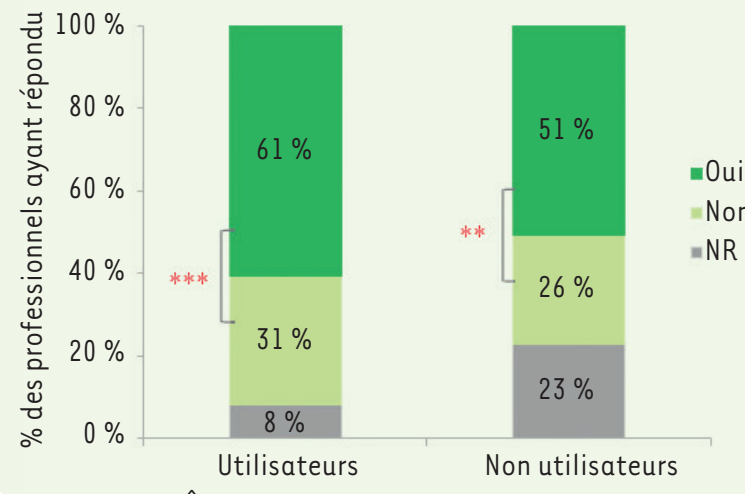

C Êtes vous suffisamment informé/formé à l'utilisation du placebo?

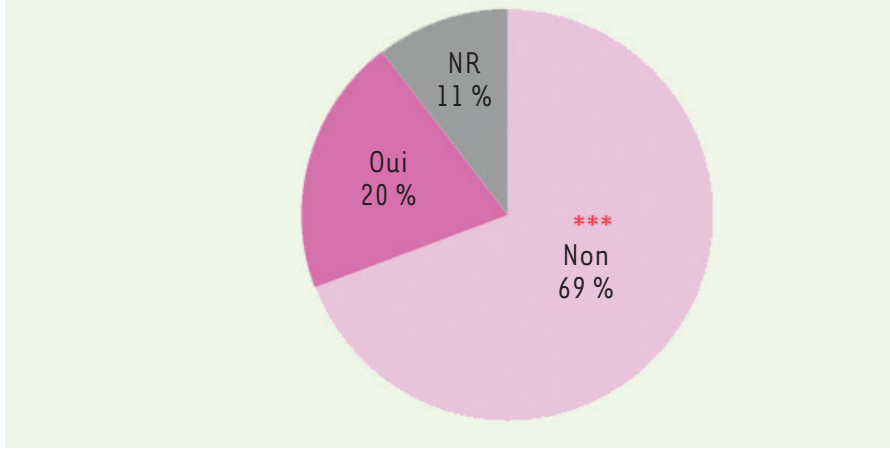

B Considérez-vous que la prescription d'un placebo influence la relation soignant-soigné?

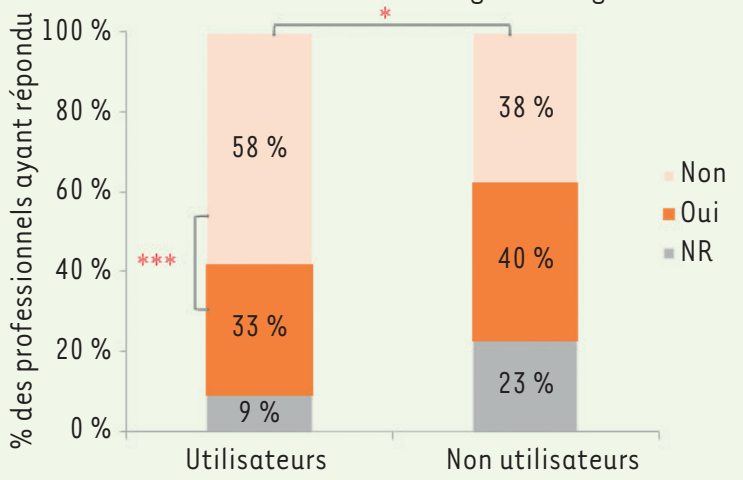

D

Croyez-vous à l'effet placebo?

Figure 2. Opinions des professionnels de santé des unités de médecine polyvalente, utilisateurs et non utilisateurs de placebo. A. Opinion sur l'efficacité du placebo. B. Opinion sur l'influence du placebo dans la relation soignant/soigné. C. Pourcentage des professionnels se considérant insuffisamment informés/formés à l'utilisation du placebo. $D$. Pourcentage de professionnels « croyant » en l'effet placebo. ${ }^{\star} p<0,05,{ }^{\star} \star_{p}<0,01$, $\star * \star p<0,001$ degré de significativité des différences inter-groupes. NR : non renseigné.

thérapeutique. Par le placebo, ils imaginent éviter le risque d'interactions médicamenteuses. Administrer un anxiolytique à une personne âgée polypathologique, en particulier lorsqu'elle prend déjà plusieurs médicaments, peut se révéler problématique. Le placebo constitue alors un traitement «miracle » et les professionnels estiment qu'ils ne prennent aucun risque en l'utilisant. Pourtant, l'utilisation du placebo n'est pas dénuée de risques puisqu'elle peut parfois entraîner des effets indésirables. Mais les professionnels sont-ils conscients de ce risque quand ils utilisent un placebo?

\section{Prescription médicale et information du patient}

La majorité des professionnels de médecine polyvalente, utilisateurs ou non du placebo, considèrent que le placebo peut constituer un traitement efficace. Néanmoins ceux qui ne l'utilisent pas craignent que sa prescription puisse influencer la relation entre soignant et patient, en particulier si le patient découvre le «subterfuge ».

Le choix d'utiliser un placebo ne devrait être fait qu'après une prescription médicale, le placebo étant considéré comme une préparation hospitalière. Donner un placebo doit également être mentionné dans le dossier médical du patient. Les patients devraient aussi être systé- matiquement informés avant de recevoir un placebo. En pratique, ils ne le sont presque jamais.

On pourrait supposer que quand un patient sait qu'il reçoit un placebo, l'effet placebo en est affecté. Plusieurs études récentes suggèrent cependant que dire au patient qu'il reçoit un placebo avant son administration ne réduit pas son effet. Des explications sur les effets et les mécanismes d'action d'un placebo données au patient se révèlent efficaces pour réduire des symptômes douloureux, notamment ceux du côlon irritable, des rhinites et des lombalgies chroniques [34-37]. Ces placebos connus du patient sont qualifiés de «placebos ouverts ». Ils contribuent au bien-être global du patient hospitalisé. Donner une information préalable au patient permettrait aux professionnels d'être en accord avec les recommandations de la Haute autorité de santé (HAS) en matière de pratiques médicales.

Notre enquête révèle que la majorité des professionnels de santé hospitaliers déclarent être insuffisamment informés. Une formation sensibilisant les professionnels à 


\begin{tabular}{lccc}
\hline Profession & $\begin{array}{c}\text { \% du nombre total } \\
\text { de réponses }\end{array}$ & $\begin{array}{c}\text { Moyenne d'âge } \\
\pm \text { Écart type }\end{array}$ & $\begin{array}{c}\text { \% d'utilisateurs } \\
\text { de placebo }\end{array}$ \\
\hline Aide-soignant(e) & $9 \%$ & $42,3 \pm 11,1$ & $43 \%$ \\
\hline Infirmier(e) & $27 \%$ & $37,8 \pm 10,0$ & $78 \%$ \\
\hline Cadre de santé & $5 \%$ & $46,5 \pm 6,2$ & $75 \%$ \\
\hline Interne & $4 \%$ & $26,6 \pm 1,3$ & $67 \%$ \\
\hline Médecin & $55 \%$ & $44,3 \pm 9,6$ & $62 \%$ \\
\hline
\end{tabular}

Tableau II. Pourcentage de professionnels qui ont répondu à l'enquête.

I'utilisation raisonnée du placebo, conformément aux recommandations de la pratique clinique, pourrait être mise en place. II reste également nécessaire de réaliser une étude quantitative sur les prescriptions des pharmacies hospitalières, afin de préciser la nature et les quantités réelles de placebos qui sont utilisés par les professionnels de santé en France.

\section{Conclusions}

À notre connaissance, aucune enquête n'a encore été réalisée dans les services hospitaliers français concernant l'usage du placebo. Notre enquête remet ainsi en question certaines pratiques des services hospitaliers dans I'utilisation du placebo à des fins thérapeutiques, notamment dans la prise en charge des patients poly-pathologiques.

Bien que l'effet placebo ait été démontré de façon incontestable, un traitement par placebo doit être utilisé avec prudence et discernement. $\varepsilon n$ aucun cas, et pour des raisons éthiques et légales, un traitement placebo ne peut remplacer un traitement conventionnel dont l'efficacité thérapeutique est prouvée. II y aurait alors une «perte de chance» pour le patient. Le placebo doit être réservé à une pratique médicale de confort et non à une pratique médicale d'urgence car il est sans garantie de résultat et peut avoir des effets indésirables.

Au vu des données récentes de la littérature, les placebos «ouverts 》 administrés en toute transparence continueraient d'être actifs chez les patients. Dès lors, il convient en pratique clinique de prendre le temps d'expliquer au patient les effets et les mécanismes d'action du placebo. À la lumière de notre étude, il apparaît essentiel de former les professionnels de santé à l'utilisation du placebo, en insistant sur la nécessité d'une prescription avec traçabilité dans le dossier médical, et d'une information préalable du patient visant à potentialiser les effets bénéfiques du placebo. $\diamond$

\section{SUMMARY}

Use of placebo in French hospitals: data from polyvalent medicine units A placebo drug is defined as a treatment without any specific pharmacological efficacy, that works when the patient thinks to receive an active treatment, through a psychological and physiological mechanism. This study aimed to evaluate the use of placebo in French hospitals, in
Polyvalent Medicine units. A questionnaire comprising 15 items was sent to 372 units. The analysis of 153 responses was conducted from dynamic crosstabs in Excel and using the $\mathrm{R}$ software available online. The survey confirmed that the use of placebos in hospital is frequent, with nearly $2 / 3$ of professionals answering the questionnaire declared to use it. The oral capsule is the most commonly used form. Placebo is mainly administered at night, in case of pain, insomnia or anxiety, to so-called "difficult" patients. Placebo is not always given after medical prescription. In most cases, patients are not informed that they receive a placebo. The majority of professionals believed in the placebo effect but considered to be insufficiently informed and trained in the use of placebo in current practice. Although the placebo effect is now demonstrated, ethical and legal considerations recommend placebo treatment only on medical prescription, with the prior information of the patient. The placebo could be used as complementary therapy to conventional treatment in the cases of this therapeutic effectiveness has been demonstrated. Professionals should be trained in the use of placebo in order to avoid nocebo effect and potentiate beneficial effects of placebo. $\diamond$

\section{REMERCIEMENTS}

Les auteurs remercient Alison Stocchetti pour sa participation à l'analyse statistique des données, ainsi qu'Odile Maillet pour la mise en forme du questionnaire.

\section{LIENS D'INTÉRÊT}

Les auteurs déclarent n'avoir aucun lien d'intérêt concernant les données publiées dans cet article.

\section{RÉFÉRENCES}

1. Dodd S. Current insights in the placebo and nocebo phenomena. Clin Ther 2017 ; 39 : 456-7.

2. Rief W, Bingel U, Schedlowski M, Enck P. Mechanisms involved in placebo and nocebo responses and implications for drug trials. Clin Pharmacol Ther $2011 ; 90: 722-6$. 


\section{RÉFÉRENCES}

3. Hahn RA. The nocebo phenomenon: concept, evidence, and implications for public health. Prev Med $1997 ; 26: 607-11$.

4. Wolf $S$. Effects of suggestion and conditioning on the action of chemical agents in human subjects: the pharmacology of placebos. J Clin Invest $1950 ; 29: 100-9$.

5. Autret A. Les effets placebo des relations entre croyances et médecines. Paris : L'Harmattan; 2013 : 112 p.

6. Lemoine P. Le mystère du placebo. Paris : Odile Jacob, $2006: 238 \mathrm{p}$.

7. Hróbjartsson A, Gøtzsche PC. Placebo interventions for all clinical conditions. Cochrane Database Syst Rev 2010 Jan 20 ; (1) : CD003974.

8. Colagiuri B, Schenk LA, Kessler MD, et al. The placebo effect: from concepts to genes.

Neuroscience 2015 ; 307 : 171-90.

9. Wartolowska KA, Feakins BG, Collins GS, et al. The magnitude and temporal changes of response in the placebo arm of surgical randomized controlled trials: a systematic review and meta-analysis. Trials $2016 ; 17: 589$

10. Cragg JJ, Warner FM, Finnerup NB, et al. Meta-analysis of placebo responses in central neuropathic pain: impact of subject, study, and pain characteristics. Pain 2016; $157: 530-40$.

11. Thomas KB. General practice consultations: is there any point in being positive? Br Med J Clin Res Ed $1987 ; 294: 1200-2$

12. Turner JA. The importance of placebo effects in pain treatment and research. JAMA $1994 ; 271$ : 1609.

13. Meissner K, Fässler M, Rücker G, et al. Differential effectiveness of placebo treatments: A systematic review of migraine prophylaxis. JAMA Intern Med 2013; 173 : 1941.

14. Kaptchuk TJ. Placebo studies and ritual theory: a comparative analysis of Navajo, acupuncture and biomedical healing. Philos Trans R Soc Lond B Biol Sci $2011 ; 366$ : 1849-58.

15. Kerr CE, Shaw JR, Conboy LA, et al. Placebo acupuncture as a form of ritual touch healing: a neurophenomenological model. Conscious Cogn 2011; 20 : 784-91.

16. Geuter S, Koban L, Wager TD. The cognitive neuroscience of placebo effects: Concepts, predictions, and physiology. Annu Rev Neurosci 2017 ; 40 : 167-88.

17. Wager TD. Placebo-induced changes in $\mathrm{FMRI}$ in the anticipation and experience of pain. Science $2004 ; 303: 1162-7$

18. Schafer SM, Colloca L, Wager TD. Conditioned placebo analgesia persists when subjects know they are receiving a placebo.J Pain $2015 ; 16: 412-20$.

19. de la Fuente-Fernandez R. Expectation and dopamine release: mechanism of the placebo effect in Parkinson's disease. Science $2001 ; 293: 1164-6$.

20. Hall KT, Lembo AJ, Kirsch I, et al. Catechol-0-methyltransferase val158met polymorphism predicts placebo effect in irritable bowel syndrome. PLoS One $2012 ; 7$ : e48135.

21. Tacheau M, Reny J, Crépin $\varepsilon$, et al. Le placebo a-t-il sa place dans la relation soignant-soigné à l'hôpital ? Éthique et Santé 2017 ; $14: 4-10$.

22. Hawke K, van Driel ML, Buffington BJ, et al. Homeopathic medicinal products for preventing and treating acute respiratory tract infections in children. Cochrane Database Syst Rev 2018 ; 9 : CD005974.

23. Sherman R, Hickner J. Academic physicians use placebos in clinical practice and believe in the mind-body connection. J Gen Intern Med 2008 ; $23: 7-10$.
24. Berthelot JM, Maugars Y, Abgrall M, Prost A. Interindividual variations in beliefs about the placebo effect: a study in 300 rheumatology inpatients and 100 nurses. It Bone Spine Rev Rhum $2001 ; 68: 65-70$.

25. Fässler M, Meissner K, Schneider A, Linde K. Frequency and circumstances of placebo use in clinical practice: a systematic review of empirical studies. BMC Med $2010 ; 8: 15$.

26. Haour F. Mécanismes de l'effet placebo et du conditionnement : Données neurobiologiques chez l'homme et l'animal. Med Sci (Paris) $2005 ; 21: 315$

27. Bouhassira D. L'anti-douleur. Paris : Le Cherche-Midi, 2018 : 208 p.

28. Guess HA. The science of the placebo: toward an interdisciplinary research agenda. Londres: BMJ Books, $2002: 332 \mathrm{p}$.

29. Kong J, Spaeth R, Cook A, et al. Are all placebo effects equal? Placebo pills, sham acupuncture, cue conditioning and their association. PLoS One 2013 $8:$ e 67485

30. Finniss DG, Benedetti F. Mechanisms of the placebo response and their impact on clinical trials and clinical practice. Pain $2005 ; 114: 3-6$.

31. Vital Durand D, Fassier T. Peut-on étudier scientifiquement le placebo? Médecine Thérapeutique $2010 ; 16: 252-8$.

32. de Craen AJ, Tijssen JG, de Gans J, Kleijnen J. Placebo effect in the acute treatment of migraine: subcutaneous placebos are better than oral placebos. J Neurol $2000 ; 247: 183-8$.

33. Kaptchuk TJ, Goldman P, Stone DA, Stason WB. Do medical devices have enhanced placebo effects? J Clin Epidemiol $2000 ; 53$ : 786-92.

34. Schaefer M, Sahin T, Berstecher B. Why do open-label placebos work? A randomized controlled trial of an open-label placebo induction with and without extended information about the placebo effect in allergic rhinitis. PLoS One 2018 ; 13 : e0192758.

35. Locher C, Frey Nascimento A, Kirsch I, et al. Is the rationale more important than deception? A randomized controlled trial of open-label placebo analgesia. Pain $2017 ; 158: 2320-8$.

36. Kaptchuk TJ, Friedlander $\varepsilon$, Kelley JM, et al. Placebos without deception: a randomized controlled trial in irritable bowel syndrome. PLoS One $2010 ; 5$ : el559l.

37. Carvalho C, Caetano JM, Cunha L, et al. Open-label placebo treatment in chronic low back pain: a randomized controlled trial. Pain 2016 ; 157 : 276672.

38. Jordan B. Cancer : thérapies « complémentaires 》 et observance du traitement anti-tumoral font-elles bon ménage ? Med Sci (Paris) 2018 ; 34 999-1002.

\section{Étudiants en master, un soutien à la recherche du Comité pour l'histoire de l'Inserm 2019-2020}

Pour l'année universitaire 2019-2020, le Comité pour l'histoire de l'Inserm propose un soutien financier aux étudiants de master qui s'engageront dans un mémoire de recherche concernant l'histoire de l'institution ou, plus largement, l'histoire de la recherche biomédicale et des questions de santé.

Les candidats, historiens ou étudiants relevant d'une autre discipline mais intégrant une approche historienne, peuvent élaborer, avec l'aide d'un directeur de recherche, leur propre sujet. Un livret de présentation, avec des propositions de sujet, est disponible.

\section{La date limite de réception des dossiers de candidature est le 18 octobre 2019}

Pour toute information, consultez le site Inserm (https://www.inserm.fr) et contactez le secrétariat scientifique du Comité : celine.paillette@ext.inserm.fr»

Pour télécharger le livret, suivez ce lien : https://www.inserm.fr/actualites-et-evenements/actualites/soutien-recherche-surhistoire-recherche-biomedicale-appel-2019-2020 